\title{
La cooperación al desarrollo como actor en la gestión turística de los espacios urbanos y patrimoniales: el estudio de caso de la AECID en América Latina Development cooperation as an actor in the tourism management of urban and heritage spaces: AECID case study in Latin America
}

\author{
Miriam Menchero Sánchez \\ (iD) https://orcid.org/0000-0001-8827-4839 \\ Universidad Internacional de La Rioja, España. \\ miriam.menchero@unir.net
}

\section{Resumen}

El tercer sector y las agencias de cooperación participan, cada vez más, dentro de la gestión de los destinos, aunque no formen parte del sistema turístico de forma estricta. Atendiendo a esta relación, apenas estudiada, el siguiente artículo analiza a la Agencia Española de Cooperación Internacional para el Desarrollo (AECID) como posible actor en el desarrollo turístico de los centros históricos latinoamericanos en los que ha intervenido a través de su emblemático Programa de Patrimonio. Para ello se ha realizado una profunda revisión bibliografía y documentación institucional, y se han entrevistado a fuentes primarias vinculadas. Todo lo anterior, para extraer conclusiones que permitan señalar en qué modo se está produciendo sinergias y se establecen algunas pautas de refuerzo en este novedoso ámbito de las relaciones hispanoamericanas.

Palabras clave: Latinoamérica, centros históricos, turismo cultural, cooperación internacional, España.

\section{Abstract}

The third sector and development cooperation agencies participate, increasingly, in the management of touristic destinations. This article analyzes this relationship barely studied through the Spanish Agency for International Development Cooperation and its Program of Cultural Heritage in Latin American historic centers. For this, different bibliography and institutional documentation have been analyzed and several stakeholders of interest have been interviewed. Conclusions will be presented that indicate in what way synergies are being produced, as well as establishing guidelines to reinforce these Spanish-American relations.

Key words: Latin America, historic centres, cultural tourism, international cooperation, Spain.

\section{Sumario}

1. Introducción |2. Centros históricos coloniales, turismo y (cooperación al) desarrollo |3. Metodología |4. Resultados | 4.1. Turismo y cooperación española en patrimonio desde sus actuaciones | 4.2. Turismo y cooperación española en patrimonio desde sus impactos | 4.3. El turismo desde la visión institucional de los actores intervinientes | 5. Conclusiones | Referencias bibliográficas

\section{Cómo citar este artículo}

Menchero, M. (2021): "La cooperación al desarrollo como actor en la gestión turística de los espacios urbanos y patrimoniales: el estudio de caso de la AECID en América Latina", methaodos. revista de ciencias sociales, 9 (1): 60-76. http://dx.doi.org/10.17502/mrcs.v9i1.442 


\section{Introducción}

América Latina posee un rico y diverso patrimonio cultural, tal y como se evidencia en el hecho de que, sobre un total de 140 bienes inscritos en la Lista de Patrimonio Mundial, 97 sean de tipología cultural y otros 38 de carácter mixto (UNESCO, 2014). A su vez, el turismo cultural se basa en el rendimiento económico de los recursos culturales y patrimoniales, llegando a suponer casi un $40 \%$ de los ingresos turísticos mundiales (OMT, 2005). Dentro de estas prácticas turísticas, el patrimonio cultural urbano, es decir, el vinculado a las ciudades, se alza, además de como un atractivo primordial para el turismo, como un motor de desarrollo sociocultural.

No obstante, se desconoce el número exacto de ciudades históricas conservadas en la región, y su concreción es motivo de disputa por parte de diferentes autores. Mientras algunos incluyen en este concepto a las urbes precolombinas (Gutiérrez, 2014), otros lo constriñen solo a espacios creados por la colonización española y lusa (De Terán, 2004). Sea como fuere, los siglos XVI y XVII supusieron un periodo de intensa urbanización en la región, pues como sostiene Brewer-Carias: "Ningún país [España] ha fundado tantos pueblos, villas y ciudades en un territorio tan grande, en un periodo de tiempo tan corto, y de una forma tan regular y ordenada" (2008: 24).

En efecto, la creación de ciudades se instrumentalizó por la corona española como un método de conquista y de división administrativa del territorio incorporado. Su vasta localización supuso una especialización funcional de algunas urbes, 1 ) bien para crear un sistema portuario a través de lugares como Veracruz, Cartagena de Indias, Portobello, Lima o La Habana, entre otras, amurallándose en la mayoría de los casos para protegerse de las incursiones foráneas; 2) bien para la explotación minera, tanto en México con Guanajuato o Potosí en Bolivia. Además, a partir de 1573, se crean las Ilamadas "Leyes de Indias", que son un detallado decálogo urbanístico que establecía la plaza mayor o de armas como origen fundacional y centro del gobierno civil y religioso, conformando un espacio de alta concentración arquitectónicopatrimonial (Gutiérrez, 2014) (Mapa 1).

Mapa 1. Centros históricos coloniales más representativos y fecha fundación

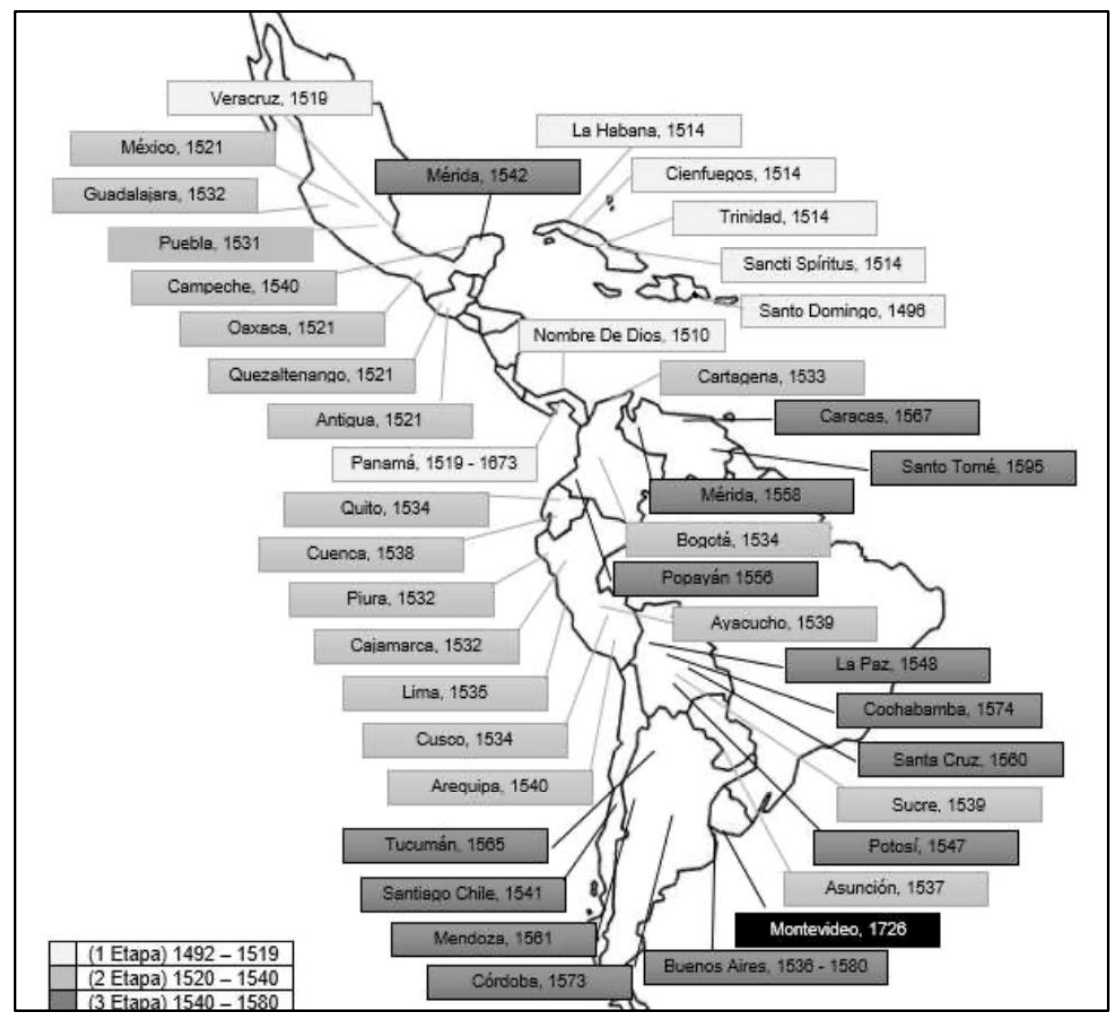

Fuente: Elaboración propia a partir de Hardoy y Tovar (1969). 
Con los procesos de emancipación muchas de estas urbes reforzaron su papel e importancia como nodos, incorporando influencias externas, como es el caso de Buenos Aires, Santiago de Chile y la mayor parte de las capitales actuales del continente. Otras, por el contrario, quedaron anquilosadas durante largos periodos de tiempo (Chueca-Goitia, 1968). Durante el siglo XX, muchas tuvieron que confrontar diferentes hechos sociopolíticos, como un profundo proceso de industrialización y urbanización de la región y una serie de catástrofes naturales, especialmente, en forma de sismos. Todo lo anterior propició que muchos de estos centros hayan establecido programas, acciones y proyectos de revitalización urbana de distinta índole. En algunos casos, la inversión ha sido tan elevada, y en cambio, la capacidad de las instituciones locales y/o nacionales tan mermadas, que se han encontrado en la necesidad de solicitar ayuda internacional incluyendo la petición de fondos para la conservación de un patrimonio cultural urbano que, en ocasiones, acusaba una alta degradación y deterioro (Muntal, 2003).

A partir de esta coyuntura, serán numerosas las entidades internacionales y bilaterales que intervendrán hasta tal punto, que la cooperación internacional se convertirá, desde la segunda mitad del siglo $X X$, en "uno de los mecanismos técnicos y financieros que han intervenido e intervienen en el patrimonio cultural de los centros históricos de América Latina (...) [y que] se la puede considerar como un actor patrimonial" (Menchero, 2019: 100). En todo caso, el objetivo de este articulo no es determinar el papel de la cooperación en la gestión patrimonial, sino su vinculación a partir de esta como actor del sistema turístico de los centros históricos latinoamericanos. Para ello y entre otros organismos, se ha seleccionado a la cooperación española y su Programa de Patrimonio Cultural, en tanto que se trata del ente bilateral que mayores fondos ha destinado a la labor de recuperación del patrimonio cultural urbano latinoamericano.

\section{Centros históricos coloniales, turismo y (cooperación al) desarrollo}

Las ciudades históricas conforman uno de los destinos más antiguos y arraigados en Europa, cuyo origen puede remontarse al Grand Tour (Prats, 1997; Ashworth, 1999). A pesar de su longeva trayectoria, durante el siglo XX fueron perdiendo protagonismo e importancia frente a otras tipologías turísticas (González y Morales, 2009). Así, en la década de los 90, se produce un resurgimiento turístico de los centros históricos, lo que supone un reconocimiento que muchas de ellas experimentan por medio de sus declaratorias nacionales e internacionales (Richards, 2008). De hecho, la propia Organización Mundial del Turismo (OMT, 2005) llega a reconocer el posicionamiento de nuevas ciudades ubicadas en países de menor proyección turística internacional.

En la actualidad, ciudades históricas, como Cartagena de Indias, Cuzco, Antigua o La Habana, reconocen y potencian el valor turístico de su patrimonio cultural urbano. Sin embargo, hasta hace cien años apenas se evidenciaban visitas puntuales a algunas capitales latinoamericanas como Buenos Aires o Lima. La ralentización de los flujos turísticos a estos espacios históricos durante toda la primera mitad del siglo XX se justifica por los altos costes y riesgos que implicaban, y solo a partir de 1960 se intensifican en ciudades como La Habana o Cuzco (Schlüter, 1998). Diez años más tarde y coincidiendo con una época de intervencionismo urbano en ciertos monumentos coloniales, se mejorará el atractivo de ciudades como Cartagena de Indias, Lima, Guatemala, Antigua, Quito, Ouro Preto o Salvador de Bahía, entre otras. No obstante, la activación turística de otros centros no se producirá ya hasta llegados los años 90, cuando se incorporan al circuito cultural internacional ciudades como Santo Domingo, Colonia de Sacramento o Ciudad de Panamá (Jordan y Duval, 2009). El aperturismo hacia destinos no globalizados y el incremento del turismo interno regional, entre otros factores, finalmente permitirá incorporar, ya en el presente siglo, a otras ciudades como Puebla, Oaxaca, Guanajuato, Potosí o Sucre, entre otras (Tresserras, 2002).

Los centros históricos son la principal manifestación del turismo urbano en Latinoamérica, y cada vez más, se prioriza su función recreativa y turística en detrimento de otras (Hiernaux, 2015). El reconocimiento turístico de estos espacios viene motivado, principalmente, por su arquitectura y urbanismo, aunque también se aprecia un interés creciente del patrimonio inmaterial vinculado con el folklore y la gastronomía local. En el ámbito inmueble, los focos turísticos se concentran en los conjuntos monumentales que forman las ya citadas plazas centrales y los enclaves religiosos coloniales (López, 1999). Aunque estos espacios nunca se diseñaron con una pretendida opulencia, parece ser que la impronta colonial produce una gran sugestión en los visitantes (Gutiérrez, 2014). 
Las áreas históricas constituyen las áreas más visitadas de las ciudades históricas (Ashworth, 1999). Son realidades vivas y por tanto implican una gran complejidad en la gestión y ordenación urbana, además de producir ciertos efectos que conviene controlar (Prats, 1997). Así lo demuestran algunos impactos que se han visualizado en ciertas ciudades históricas latinas. Por un lado, destacan, en el aspecto urbano, el deterioro de espacios o infraestructuras públicas ocasionados por problemas de tránsito vehicular o peatonal. También se produce contaminación acústica o visual, tal y como sucede en Cartagena de Indias, Viejo San Juan, Panamá o La Habana (Sanz, 1996). Respecto a los efectos socioculturales, se ha manifestado una recuperación de la identidad, o, en sentido contrario, una banalización cultural, como ocurre en Taxco, San Miguel de Allende, o en ciudad andinas como Cuzco (Sanz, 1996).

Estas dinámicas pueden tener una incidencia en el patrimonio cultural inmueble, ya que en ocasiones se producen "escenografías turísticas" al intervenir sobre los usos tradicionales de los edificios por fines exclusivamente turísticos o de especulación inmobiliaria - tal y como ya ha sucedido en Cartagena de Indias, Panamá o Antigua Guatemala (Ballart, 2002)-. Estas circunstancias pueden derivar en procesos de "museificación", en los que se prioriza la función turística del espacio urbano en detrimento de otras como ha podido suceder con Colonia de Sacramento (Gutman y Hardoy, 1992). Muy vinculado a lo anterior, pueden proliferar ciertas franquicias -como un Starbucks en la plaza de armas de Cuzco- reemplazando establecimientos gastronómicos locales o de artesanías por tiendas de recuerdos estandarizados (Pulin, 1993).

Sea como fuere, no existe un consenso sobre los efectos turísticos generados en los centros históricos coloniales, aunque sí existe un acuerdo en imputar estos impactos a la insostenibilidad de un modelo de desarrollo turístico masivo (Orbasli, 2002). Según la UNESCO (2014), este es el gran reto que presentan actualmente las ciudades históricas latinoamericanas: no enfatizar el aspecto estético-colonial en detrimento del papel y función original de los centros históricos.

Por otra parte, cualquier destino, con indiferencia de su escala o tipología, reúne a una serie de actores vinculados directa e indirectamente con la actividad turística. En el caso de los centros históricos, las instituciones encargadas de la gestión del patrimonio cultural componen uno de los grupos de mayor importancia ${ }^{1}$. Como se señalaba al inicio, dentro de estas relaciones se pueden incluir a las agencias internacionales de cooperación, dado que a veces actúan como

[U]na herramienta ideal para la puesta en valor de los recursos de los territorios menos desarrollados o en desarrollo. Los recursos culturales tienen un enorme potencial de producción turística y generan interés, de ahí la necesidad de incluir también en este análisis a este tipo de agencias (Pulido, 2015: 151).

Además de la capacidad para dotar de funcionalidad turística al patrimonio cultural, existen agentes del tercer sector que pueden asumir funciones similares a la gestión turística, especialmente en los países menos desarrollados. Dentro de este grupo, se aprecia una amplia heterogeneidad de actores -asociaciones, organizaciones públicas, privadas, con o sin fines de lucro, voluntariado o instituciones internacionales de crédito, entre otras-. También abarcan una variada y cada vez más compleja y difusa temática, a la que hay que sumar la propia transversalidad del turismo y del patrimonio cultural (Morère y Perelló, 2012). De este modo, la cooperación en turismo no necesariamente procede de la asistencia internacional sectorial, y puede hacerlo desde otros ámbitos urbanos. Como señala Pulido: "Cada vez es más común encontrar intervenciones destinadas a poner en valor el patrimonio de una localidad o territorio y a que estas intervenciones se hagan en nombre del desarrollo turístico del lugar" (2015: 151).

Bajo esta premisa se puede encontrar todo un despliegue normativo y de intervención en los centros históricos latinoamericanos. Primeramente, se puede verificar la existencia de diferentes cartas internacionales y regionales que avalan la ayuda internacional en los centros históricos, a la par que señalan la valoración turística de estos espacios urbanos. Al respecto, la reconocida Convención de 1972 para la Protección del Patrimonio Mundial Cultural y Nacional (UNESCO), no solo recomienda la necesaria colaboración entre países miembros en el ámbito, sino que establece el desarrollo de todo un aparato institucional para la captación financiera a través del Fondo de Patrimonio Mundial. De igual manera, la Carta Internacional para la Conservación de Ciudades Históricas y Áreas Urbanas Históricas, también Ilamada Carta

\footnotetext{
${ }^{1}$ Los propietarios y/o gestores culturales tienen un papel primordial en la adecuación turística del patrimonio, lo que requiere una coordinación y colaboración por su parte con el ámbito turístico, aunque en ocasiones ambos presenten enfoques diversos o contradictorios (Ashworth, 1999).
} 
de Washington (1987, ICOMOS), reincide en la financiación de los espacios urbanos declarados a la vez que es la primera en mencionar los efectos del turismo asociados mayormente a los casos de "explotaciones turísticas excesivas" del patrimonio urbano (Capitulo IV).

En el ámbito latinoamericano, son las Normas de Quito (OEA, 1967) las que despliegan todo un espíritu de cooperación interamericana, por medio de tres premisas: 1) la grave situación de los centros históricos de la región, 2) el uso del patrimonio cultural como instrumento de desarrollo, y 3) el turismo como aliado de la recuperación monumental urbana (González Varas, 1999). De este modo, la incapacidad de los países para afrontar acciones para la salvaguardia patrimonial se suma a la justificación de intervención y adecuación de los monumentos para el incremento del turismo -e incluso para la mejora del sentido de pertenencia de sus residentes (Arts. 7.4, 7.5 y 7.1.). Diez años más tarde, un nuevo Coloquio de Quito (1977, UNESCO-PNUD) cuestionará tales lineamientos, al criticar ciertos "maquillajes escenográficos" en aras de una finalidad excesivamente turística. Es por ello por lo que esta nueva normativa buscará un desarrollo urbano más amplio, sin eliminar al turismo, pero con una priorización de las acciones sociales y de la dotación de infraestructuras asistenciales (González Varas, 1999).

Existe, además, una evidente plasmación de estos lineamientos internacionales y regionales en numerosos proyectos de cooperación internacional en el patrimonio cultural urbano latinoamericano. En primer lugar, en los años 50, la UNESCO desarrolló un programa de restauración monumental bajo el paraguas de la llamada "solidaridad" internacional, el cual tuvo un amplio alcance en la región, con ciudades beneficiadas como Cuzco (1950), La Paz, Oruro, Potosí (1966), Cartagena de Indias (1968) y Santo Domingo (1968) (UNESCO, 1969). Aunque no se puede evidenciar una relación directa con el turismo, en la actualidad y por medio del Centro de Patrimonio Mundial, la UNESCO -en colaboración con otras entidades de la ONU - sigue manteniendo acciones prioritarias sobre el Patrimonio Mundial Latinoamericano con la finalidad de establecer sinergias con el sector (UNESCO, 2014).

Por otra parte, pese a la amplia labor de acompañamiento y asesoramiento del organismo, los autores coinciden en señalar a otras instituciones y programas como actores participantes. A tal efecto, el Programa para el Desarrollo de las Naciones Unidas (PNUD) (Carrión, 2007) ha desarrollado desde la década de los años 70 diferentes iniciativas en el ámbito. Uno de sus proyectos más emblemáticos será la puesta en funcionamiento del Plan de Desarrollo Turístico Integral (Plan COPESCO). Este proyecto, basado en la preservación y uso del patrimonio cultural de la ciudad de Cuzco buscaba mejorar la calidad de vida de su población por medio de la inversión en infraestructura y turismo. De esta manera, se restauraron y acondicionaron para la visita turística hasta un total de 27 monumentos arqueológicos y coloniales (BID, 2001).

Posteriormente, desde la década de los 90, el PNUD ha desarrollado otros proyectos similares. Por medio de la intervención "Fortalecimiento económico y cultural de La Habana" (1995-2005), se buscó la revitalización del centro a través de aspectos habitacionales, sanitarios y educativos, pero también el apoyo y promoción a servicios turísticos sostenibles. Así mismo, a través del proyecto "Recuperación y puesta en valor de la ciudad histórica de Panamá (2012-2016)" se ahonda en la relación entre la rehabilitación patrimonial y el uso turístico. En este último caso, además, se buscó una inclusión, participación y apropiación de la comunidad residente y de las entidades locales interesadas.

El otro gran financiador es el Banco Interamericano de Desarrollo (BID) que, como entidad de crédito de la región, se compromete a la revitalización del patrimonio urbano y al fomento del turismo cultural durante el último tercio del siglo XX. Uno de sus proyectos más emblemáticos recae sobre la ciudad de Quito (1994), con el fin de hacer frente a la progresiva degradación de su zona histórica. Además del BID, se contó con la participación de la UNESCO, el PNUD y socios bilaterales como España o Bélgica. Aunque una partida importante de esta iniciativa se destinó a la infraestructura urbana, otras líneas incidieron en la dotación cultural y patrimonial, y el fomento a la inversión para proyectos comerciales y turísticos (BID, 2001). El otro gran proyecto fue el programa "Monumenta", enfocado a 46 centros históricos brasileños en situación de deterioro. Muchos de ellos, como Recife y Ouro Preto, fueron intervenidos con una clara finalidad recreativa y turística, incidiendo en mejorar los espacios patrimoniales y públicos (BID, 2001). El éxito de este programa lo convirtió en un icono y conllevó iniciativas similares, aunque de menor envergadura como: 1) el plan de intervención del centro histórico de Santo Domingo" (2011 - 2018) -que incluía instrumentos de gestión turística- o 2) el proyecto integral de rehabilitación del centro histórico de Tegucigalpa (2000), en el que se determinaron usos turísticos en el espacio urbano intervenido. 
La financiación de proyectos en centros históricos latinoamericanos por actores internacionales de cooperación sigue estando vigente, especialmente en el Caribe y Centroamérica (UNESCO, 2014). A ello se le suma una reciente consideración del turismo como mecanismo para el desarrollo por parte de los organismos de cooperación (Nel-lo y Pérez, 2015). Así, a iniciativas promovidas por la OMT como el ProPoor Tourism (PPT) o el Programa "Turismo Sostenible-Eliminación de la pobreza" (ST-EP) se le debe añadir la inclusión de este ámbito económico en la consecución de los Objetivos de Desarrollo Sostenible (Gascón y Cañada, 2005). De hecho, aunque el turismo contribuye directamente a los objetivos 8, 12 y 14, también podría incluirse en el 11, que versa sobre las ciudades y sobre las que señala que,

El turismo sostenible tiene la capacidad de mejorar las infraestructuras urbanas y la accesibilidad universal, de promover la regeneración de áreas en decadencia y de preservar el patrimonio cultural y natural, activos de los que depende el turismo. Una mayor inversión en (...) conservación de los sitios patrimonio (...) debería dar como resultado unas ciudades más inteligentes de las que podrían beneficiarse no solo sus habitantes, sino también los turistas (OMT, 2015:4).

Atendiendo a ello, queda patente la vigencia actual de las iniciativas que inciden en el patrimonio cultural urbano de la región, pero igualmente los nuevos retos que la cooperación internacional presenta en los centros históricos si aspira a que el turismo se erija como un verdadero motor de desarrollo socioeconómico del continente.

\section{Metodología}

La introducción refleja que las relaciones entre patrimonio urbano y turismo han estado presentes tanto en la normatividad como el desarrollo de proyectos específicos por parte de organismos internacionales como la UNESCO o el BID. En este sentido, conviene redundar que el objetivo principal de este articulo no es el análisis de la cooperación en los centros históricos, sino su papel como actor turístico. Para ello se ha analizado el estudio de caso de la Agencia Española de Cooperación al Desarrollo (AECID) y su emblemático Programa de Patrimonio Cultural y Escuelas Taller en la región. Un proyecto vigente desde hace más de 30 años en Latinoamérica y que ha llegado a intervenir en más de 30 centros históricos -incluyendo algunos tan importantes como Cartagena de Indias, Cusco, La Habana, Quito, Santo Domingo, entre otros- y en más de 250 inmuebles vinculados a estos espacios urbanos (Menchero, 2019).

Para conseguir este propósito, en un primer momento se ha realizado un estudio exploratorio de las fuentes secundarias vinculadas con la difusión y comunicación institucional que ha elaborado la AECID para la transmisión e información de su Programa de Patrimonio Cultural y Escuelas Taller. Esta consulta se ha apoyado en los medios digitales con los que cuenta la institución y en los soportes físicos que albergan el archivo y biblioteca de la sede del organismo en Madrid (España), además de en diferentes países como Colombia, Ecuador y Perú. Una vez determinadas las fuentes de información se ha procedido a la identificación de las posibles referencias directa e indirectas sobre el turismo. Los resultados obtenidos se dividieron en dos ámbitos de análisis: 1) Información obtenida de los documentos marco del programa, es decir, bibliografía previa a su ejecución y cuyos datos relevantes se centran, principalmente en la articulación de objetivos, actuaciones y conceptos clave; y 2) Datos extraídos de los estudios de evaluación de los programas, es decir, documentación realizada con posterioridad y que incluye al turismo como resultado. Toda esta información ha permitido realizar un importante análisis de fuentes bibliográficas que no se había realizado con anterioridad a esta investigación. De este modo, la información obtenida compone los dos primeros epígrafes de los resultados de este artículo.

Posteriormente y para profundizar en esa mirada más práctica sobre el programa, se han elaborado diferentes entrevistas personales que componen un análisis de fuentes primarias que complementa a lo anteriormente señalado. Para ello, se ha realizado diez entrevistas, divididas en dos ámbitos: 1) de gestión patrimonial, incluyen a la UNESCO en la región latinoamericana y a distintos cargos de responsabilidad del programa de la AECID, atendiendo a varias escalas administrativas y geográficas (sede en Madrid, sedes nacionales de América Latina y Escuelas Taller locales), 2) También se entrevistó a miembros de organismos vinculados con el desarrollo turístico de los centros históricos coloniales, especialmente a entidades locales y al representante de la subregión de "las Américas" de la Organización Mundial del Turismo (Cuadro 1). 
Cuadro 1. Listado de entrevistados

\begin{tabular}{|c|c|c|c|}
\hline Ámbito & Escala & Cargo & Cuestionario \\
\hline \multirow[b]{2}{*}{ Patrimonio } & Regional & $\begin{array}{l}\text { 1. Responsable UNESCO para la región } \\
\text { andina } \\
\text { 2. Jefa adjunta del Departamento de } \\
\text { Cooperación y Promoción Cultural de la } \\
\text { AECID. } \\
\text { 3. Responsable de la unidad de apoyo del } \\
\text { programa de Escuelas Taller de la AECID }\end{array}$ & $\begin{array}{l}\text { 1. Origen e intervenciones } \\
\text { en patrimonio cultural } \\
\text { urbano. } \\
\text { 2. Reconocimiento y } \\
\text { beneficiarios de los } \\
\text { proyectos. }\end{array}$ \\
\hline & $\begin{array}{l}\text { Nacional - } \\
\text { Local }\end{array}$ & $\begin{array}{l}\text { 1. Responsable del Programa de Patrimonio } \\
\text { Cultural y Escuelas Taller de la AECID en } \\
\text { Ecuador } \\
\text { 2. Directora Escuela Taller Cuenca (Ecuador) } \\
\text { 3. Directora Escuela Taller Arequipa (Perú) } \\
\text { 4. Exdirector Escuela Taller Cartagena de } \\
\text { Indias (Colombia) } \\
\end{array}$ & $\begin{array}{l}\text { 3. Futuro y relaciones de la } \\
\text { cooperación en los } \\
\text { centros históricos. } \\
\text { 4. Efectos positivos y } \\
\text { negativos generales y } \\
\text { turísticos. } \\
\text { 5. Reflexiones personales. }\end{array}$ \\
\hline \multirow[b]{2}{*}{ Turismo } & Regional & $\begin{array}{l}\text { 1. Exdirector regional de la OMT para las } \\
\text { Américas }\end{array}$ & \multirow{2}{*}{$\begin{array}{l}\text { 1. Turismo cultural en los } \\
\text { centros históricos. } \\
\text { 2. Turismo patrimonial en } \\
\text { los centros históricos. } \\
\text { 3. Reconocimiento políticas } \\
\text { / cooperación } \\
\text { internacional. } \\
\text { 4. Uso y función atractivos } \\
\text { turísticos } \\
\text { 5. Reflexiones personales. }\end{array}$} \\
\hline & $\begin{array}{l}\text { Nacional - } \\
\text { Local }\end{array}$ & $\begin{array}{l}\text { 1. Responsable de Promoción en Quito } \\
\text { Turismo (Ecuador) } \\
\text { 2. Subgerente de Turismo de la } \\
\text { Municipalidad de Lima (Perú) } \\
\text { 3. Secretaria de Turismo y Cultura de } \\
\text { Mompox (Colombia) }\end{array}$ & \\
\hline
\end{tabular}

Fuente: Elaboración propia.

Para el primer grupo (gestores patrimoniales) se realizó un cuestionario basado en cuatro preguntas abiertas sobre las intervenciones, reconocimientos, beneficiarios y efectos turísticos del programa de la cooperación española. Para el segundo grupo (gestores turísticos), las cuatro preguntas redundaron sobre el desarrollo del turismo cultural en los centros históricos coloniales y la importancia de la cooperación internacional desde la gestión turística (Tabla 1$)^{2}$. Al respecto y por limitaciones del propio texto, se han seleccionado y escogido las respuestas que resultan más relevantes para las líneas de análisis que propone este trabajo y que han sido integradas en el último epígrafe de los resultados, y que se desarrolla a continuación.

\section{Resultados}

Como se señalaba con anterioridad, para estudiar las relaciones que se han producido entre el programa de Patrimonio de la AECID con el desarrollo turístico de los centros históricos latinoamericanos, se ha considerado su análisis desde tres ámbitos diferenciados. En primer lugar, desde su plasmación escrita a través de la documentación institucional realizada por la cooperación española en el ámbito del patrimonio urbano latinoamericano. Seguidamente, desde los informes de resultados del programa y las consecuencias directas e indirectas turísticas derivadas. Y finalmente, desde los portavoces y gestores públicos de esta relación, y que sirve para contrastar la visión literal de la producción bibliográfica con su plasmación práctica en los centros históricos latinoamericanos.

\footnotetext{
${ }^{2}$ La selección de las variables que constituyen los ejes de cada una de las preguntas de los cuestionarios realizados se obtuvo de la primera fase de la investigación, es decir, del análisis exploratorio de las fuentes secundarias. En este sentido, y dado que este artículo se inscribe en una investigación de mayor alcance, ciertas preguntas del cuestionario pueden tener más relación con la cooperación internacional en centros históricos que con la actividad turística desarrollada.
} 


\subsection{Turismo y cooperación española en patrimonio desde sus actuaciones}

Como se ha señalado en la primera parte del texto, son numerosas las entidades que han incidido en proyectos de cooperación en los cuales existe una relación entre el patrimonio urbano y el turismo cultural. En este sentido, no se han incluido aquellas relaciones bilaterales consistentes en acuerdos de colaboración entre dos países en la materia. Sin embargo, la labor desprendida de estas acciones ha sido de gran repercusión para la región, y, en este sentido, España se ha erigido como uno de los países que más asistencia técnica y financiera han destinado al patrimonio cultural latinoamericano (Muntal, 2003; Carrión, 2007).

Los orígenes de esta relación hay que fundamentarlos en la diplomacia cultural y en cierto paternalismo patente en la labor del Instituto de Cultura Hispánica (ICH) (1945-1976), posteriormente rebautizado como Instituto de Cooperación Iberoamericana (ICI) (1979-1988). Su visión del significado de "Iberoamérica" fomentó unas relaciones culturales basadas en el intercambio de autores y eventos fundamentalmente folklóricos (Del Arenal, 2011). No obstante, a partir de 1971, se intensificaron los convenios técnicos de cooperación con casi todos los países e incluso es posible documentar ya algunas incursiones en la rehabilitación de edificios como es el caso de las Casas de Benalcázar en Quito. A partir de la materialización de la Agencia Española de Cooperación Internacional (AECI) y, especialmente, de los actos conmemorativos del año 1992, el programa de patrimonio cultural coge fuerza, y posteriormente pasará a convivir con otras acciones de cooperación cultural como el Programa ACERCA, los Centros Culturales en el Exterior (CCE) o los Centros de Formación de la Cooperación Española (CFCE), entre otros (AECID, 2009, 2011, $2015)^{3}$.

En cualquier caso, el patrimonio cultural latinoamericano constituye un eje dentro de la cooperación internacional española, incluso mediante iniciativas incluidas en otros proyectos o unidades de la AECID (Nel-lo y Pérez, 2012). Las Escuelas Taller, no se unirán al Programa de Patrimonio Cultural hasta el año 1992, coincidiendo con el periodo en que mayor interés suscita la recuperación del patrimonio arquitectónico colonial (AECID, 1999). No obstante, su hasta ahora vigencia representa una longevidad de más de 30 años y una continuada renovación tanto en objetivos y escalas de actuación, en la que se puede observar una ampliación del concepto de patrimonio cultural y una reorientación hacia la gestión integral (Gómez-Pallete, 2014). También se puede apreciar una articulación hacia los planes directores de la AECID y hacia las nuevas actuaciones de la cooperación española (AECID, 2009) (Cuadro 2).

Respecto a los objetivos de cada etapa y su vinculación con el turismo, esta siempre se ha realizado de manera indirecta. En la primera etapa de ellas, bajo la denominación de "Programa de Preservación del Patrimonio Cultural de Iberoamérica (1984-1993)", el programa se justificaba por el propio fracaso del modelo urbano latinoamericano y el deterioro de los centros históricos, y se introducía un claro componente económico del patrimonio cultural en sus objetivos,

Recuperar el patrimonio como elemento activo a través de nuevos usos que permitan su reutilización al servicio de la sociedad (...) y convertir el patrimonio en un factor económicamente rentable, capaz de apoyar y dinamizar el desarrollo de los pueblos a través de su reutilización sostenida (Quinto Centenario, $1991 ; 20)$

En la segunda etapa del programa, bajo la denominación de "Patrimonio Cultural de la Cooperación Española" (1994-2004), apenas se realizaron modificaciones en los planteamientos anteriores. No obstante, el nuevo estatuto de la AECID y el cambio de adscripción administrativa produjeron una vinculación mayor de la cooperación cultural como instrumento de desarrollo. Por primera vez, se establecen prioridades sectoriales a partir del I Plan Director de la Cooperación Española (2001-2004), en el que se señala al patrimonio cultural por su capacidad como generador de empleo e ingresos por turismo (MAEC, 2000). Aun con todo, los objetivos de esta etapa no distan mucho de la anterior, y siguen mostrando una relación indirecta con el turismo por medio del "uso y disfrute del patrimonio, considerándolo como factor económico dinamizador del desarrollo" (AECID, 2010: 23).

Posteriormente y ya en la tercera etapa "P>D Patrimonio para el Desarrollo" (2005-2009), se realiza una actualización del programa y una nueva articulación con el II Plan Director de la Cooperación Española

\footnotetext{
${ }^{3}$ El programa ACERCA, vinculado a la capacitación y formación del sector cultural, establece diferentes estrategias como encuentros, congresos, talleres, intercambio, cursos o asesoramiento. En sus líneas de actuación se encuentran el empleo cultural en todos sus ámbitos, incluyendo el vinculado al patrimonio y al turismo cultural (AECID, 2013).
} 
(2005-2008). Así, se muestra unos objetivos más sociales mediante el impulso de "intervenciones de puesta en valor y gestión patrimonial para contribuir al desarrollo sostenible de las comunidades". También se redefine la terminología de actuación utilizada, incluyéndose la categoría de "turismo e industrias culturales" (AECID, 2010: 27). De hecho, se mantiene la importancia de las actividades económicas, destacándose el uso productivo del patrimonio cultural por medio del desarrollo turístico (AECID, 2015b).

La actual y ultima etapa, "P>D Patrimonio para el Desarrollo y Escuelas Taller" (2010-actualidad), se confirma como la implementación de los principios de la cooperación española en todas las líneas del programa, así como una nueva concordancia con los tres planes directores realizados: III (2009-2012), IV (2013-2016) y V (2018-2021). De este modo, desde el programa se recalca una gestión sostenible e integral del patrimonio cultural, en el que la conservación, el uso social y económico se redirija hacia la ciudadanía y el territorio (MAEC, 2009). De esta iniciativa se desprenden varios objetivos transversales del programa, en el que nuevamente se sigue hablando veladamente de cierto componente económico al señalar "el desarrollo local a través del aprovechamiento del patrimonio cultural (...) y su recuperación como recursos de desarrollo local" (AECID, 2015: 32). Es decir, el turismo se entiende como un elemento inscrito en el llamado "ciclo patrimonial", a saber, como una acción de aprovechamiento público a partir de la contribución privada (AECID, 2009). Esta nueva hoja de ruta para el programa sigue contemplando la búsqueda de sinergias a través de ámbitos complementarios a la cooperación internacional en patrimonio.

Como se desprende de lo anterior, la AECID ha incluido con mayor o menor manifiesto al turismo dentro de los objetivos de su programa patrimonial. Esta acción viene refrendada por el uso de contrapartes y socios afines. De esta forma, la cooperación española elaboró un convenio por el Consorcio de la Ciudad de Santiago de Compostela, no vigente actualmente, pero que buscaba el apoyo para una gestión turística sostenible. Así mismo, se ha buscado la implicación de entidades turísticas locales y nacionales como contrapartes, desde la Comisión Nacional de Turismo (Argentina), la Corporación de Turismo del Cauca (Colombia), el Instituto Guatemalteco de Turismo (Guatemala), el Instituto Hondureño de Turismo (Honduras), el Ministerio de Turismo (Nicaragua) o la Secretaría de Turismo de Paraguay (AECl, 1999).

Cuadro 2. Etapas y líneas de actuación, 1984-2016

\begin{tabular}{lll}
\hline PPPCI & 1. & Planes de Revitalización de Centros Histórico-Proyectos Piloto. \\
$1984-1993$ & 2. & Restauración de Monumentos y Bienes Muebles. \\
& 3. & Escuelas Taller (a partir de 1992). \\
\hline \multirow{2}{*}{ PPCCE } & 1. & Planes de Revitalización de Centros Histórico-Proyectos Piloto. \\
$1994-2004$ & 2. & Restauración de Monumentos y Bienes Muebles. \\
& 3. & Escuelas Taller (a partir de 1992). \\
& 4. & Inventarios de Bienes Culturales. \\
\hline & 1. & Instrumentos de planificación urbana y territorial. \\
P>D & 2. & Conservación y Restauración de Bienes Inmuebles. \\
$2005-2009$ & 3. & Escuelas Taller. \\
& 4. & Inventarios de Bienes Culturales. \\
& 5. & Recuperación del Patrimonio Inmaterial. \\
\hline & 1. & Instrumentos de planificación urbana y territorial. \\
P>D y PET & 2. & Formación y capacitación institucional. \\
$2010-$ Actual & 3. & Inventarios de Bienes Muebles. \\
& 4. & Conservación y restauración de Bienes muebles / inmuebles. \\
& 5. & Recuperación Patrimonio Inmaterial. \\
\hline Fuente: Elaboración propia a partir de AECID (2007, 2007a, 2007b, 2015).
\end{tabular}

Fuente: Elaboración propia a partir de AECID (2007, 2007a, 2007b, 2015).

La intervención y/o planificación urbana, junto con la restauración de monumentos y las Escuelas Taller han sido las líneas comunes a todas las etapas del programa (Figura 1$)^{4}$, y de las que mejor se puede

\footnotetext{
${ }^{4}$ El fortalecimiento institucional incluye aspectos como documentación, inventariado o gestión del patrimonio cultural. La planificación urbana y territorial, por su parte, puede incluir intervenciones en inmuebles habitacionales, espacios públicos
} 
desprender una lectura turística. En el primer caso, a través de los planes de revitalización urbana desarrollados, se vislumbran acciones vinculadas con el turismo en una doble vía. Mientras que en ciudades como Antigua Guatemala se señala al turismo como una causa de la transformación de los centros históricos, en otros casos, como en Potosí, Sucre, Quito, Popayán o Gracias, se le considera como una acción a promover y desarrollar (AECl, 2002).

Por otro lado, los "Planes Piloto", como iniciativas de restauración de monumentos vinculados a los instrumentos de planificación urbana, también han tenido una interferencia con el turismo, especialmente a través de la generación de instalaciones culturales con potencialidad turística. Así es que destaca la creación de museos como el Ingenio de San Marcos (Potosí), Antiguo Convento de San Francisco (Sucre), el Museo de Arqueología de Comayagua y espacios patrimoniales como la Iglesia de la Compañía de la Compañía de Jesús (Potosí), entre otros.

De carácter similar a los anteriores, pero sin relación con los planes urbanos, la línea de "Restauración de Monumentos" incide en la intervención de inmuebles con valor histórico y uso social posterior -incluyendo lo religioso, institucional, sociocultural y turístico (AECl, 2002)-. Con una tipología religiosa, muchos de los edificios intervenidos actúan hoy como atractivos turísticos como es el caso de la antigua Catedral de Cuenca (Ecuador), el Convento de San Francisco de Quito (Ecuador), el Convento de Santo Domingo de Cartagena de Indias (Colombia) o Santa Lucía en Suchitoto (El Salvador). También ejercen como iconos turísticos inmuebles con otros usos como el educativo en el caso de la Casona de San Marcos de Lima (Perú), o museísticos como el Museo Naval del Caribe de Cartagena de Indias (Colombia) el Santuario de Guápulo en Quito (Ecuador) o el Museo de Sitio Bodega y Quadra en Lima (Perú), por citar algunos de los más representativos (Menchero, 2020) ${ }^{5}$.

Por último, en el caso de las Escuelas Taller, igualmente se produce una doble relación con el turismo. En primer lugar, muchas de las intervenciones realizadas tanto para los proyectos piloto como para la restauración de monumentos se hicieron a través de prácticas laborales realizadas por parte de los estudiantes de estos centros de formación. Por otra parte, son numerosas las Escuelas Taller que han incluido en su oferta talleres o cursos vinculados con la actividad turística. Así, según los datos analizados hasta el año 2015, el turismo supuso el 4,15\% de la formación total, a los que, por su afinidad, conviene sumar un $4,85 \%$ de cursos de cocina y otro $5,66 \%$ de artesanías. En cualquier caso, son datos poco significativos si se comparan con la formación relacionada con la recuperación del patrimonio construido, que ha llegado a suponer hasta un $85 \%$ del total en algunas etapas del programa (AECID, 2015).

Sea como fuere, entre los cursos de turismo se encuentran los de guía o personal de alojamiento, y siempre han sido elaborados por Escuelas Taller situadas en entornos con mayor vocación turística, como Chiquitanía y Colca. En el caso de las artesanías, destacan los centros de orfebrería en Guatemala, Potosí y Mompox o la cerámica en Cuenca y Popayán. Las actividades gastronómicas, por su parte, se han focalizado en la región andina, especialmente en las escuelas taller ecuatoriana, la de Arequipa en Perú y la de Sucre en Bolivia (AECID, 2015).

\subsection{Turismo y cooperación española en patrimonio desde sus impactos}

Como se ha mostrado en el anterior epígrafe, el Programa de Patrimonio Cultural ha tenido una estrecha vinculación con la puesta en valor de los bienes inmuebles urbanos (AECl, 2002). El impacto de estas actuaciones ha trascendido de los valores culturales y ha incidido en su uso productivo, con resultados que han "propendido y facilitado un desarrollo turístico al amparo de la recuperación patrimonial" (AECID, 2015: 40). Estos efectos turísticos en los centros históricos latinoamericanos, además, vienen evidenciándose de

o áreas urbanas integrales. Finalmente, dentro de las líneas de actuación vinculadas con la conservación y restauración se encuentran edificios dotacionales, religiosos, enclaves arqueológicos y bienes muebles de cualquier tipología (AECID, 2015).

${ }^{5}$ En las intervenciones de inmuebles, la AECID ha actuado tanto de manera parcial como integran y, en general, se incluyen actuaciones como la reconstrucción física (fachadas, bóvedas, patios, estancias), la eliminación de añadidos y la restauración de elementos ornamentales perdidos, la inclusión de equipamientos, servicios básicos y la adecuación de espacios, como auditorios. En cualquier caso, todas ellas son llevadas a cabo con la finalidad de puesta en valor y dotación de nuevos usos. 
las primeras etapas del programa $(\mathrm{AECl}, 1998)^{6}$. No obstante, para la cooperación española el turismo es descrito como un efecto, impacto o resultado indirecto del programa de patrimonio cultural y siempre se asienta en tres aspectos; 1 ) el uso turístico a través de los nuevos usos públicos del patrimonio intervenido, 2) la generación de empleos vinculados y 3) los ingresos generados (AECID, 2011).

De este modo, atendiendo a la potenciación del uso turístico de los inmuebles, la cooperación española señala que, de un lado, muchos de los edificios intervenidos han pasado de no tener uso a convertirse en contenedores culturales, lo que ha supuesto una accesibilidad a cualquier tipo de publico. Estos proyectos también han mejorado el propio paisaje urbano por medio de la mejora de los entornos, destacando en este sentido las intervenciones sobre espacios públicos y la posible recuperación de las funciones sociales y económicas. Así, en ambos casos, se ha producido una incorporación de los bienes culturales en los circuitos turísticos de sus respectivas ciudades (AECID, 2003). Igualmente, en las evaluaciones del programa, hasta tres centros históricos se citan como espacios en los que la cooperación ha recuperado el patrimonio urbano con fines primordialmente turísticos: Granada (Nicaragua), el Valle del Colca (Perú) y Comayagua (Honduras) (AECID, 2015).

Al respecto, se aprecia un énfasis en el refuerzo institucional de la gestión turístico-patrimonial de algunos centros. Así se recoge para la ciudad peruana de Huamanga y el trabajo conjunto realizado con la alcaldía de la ciudad; o la Escuela Taller de Cartagena de Indias, designada por el Ministerio de Cultura de Colombia para la puesta en valor patrimonial y turística de los inmuebles que integran las fortalezas de la ciudad. Aunque esta designación se hizo ya fuera la gestión española, el Gobierno colombiano concedió dicha responsabilidad por la propia trayectoria que había desempeñado la Escuela Taller desde su origen (AECID, 2015).

Un caso de relevancia lo representa la ciudad boliviana de Potosí, en la cuál las propias instituciones municipales señalaron su incapacidad para la gestión cultural de su patrimonio histórico, así como una falta de fondos para intervenirlos y conservarlos. La ciudad que además se encuentra declarada desde 1987 como Patrimonio Mundial por la UNESCO, hizo un Ilamado para solicitar apoyo y asistencia técnica en el ámbito patrimonial, pero también para impulsar el turismo cultural en su centro histórico. Según la AECID, esta solicitud justificó la apertura de una Escuela Taller en la ciudad y las acciones de la cooperación española en el ámbito (AECID, 2015).

En todo caso, son múltiples las soluciones que el programa de patrimonio cultural ha dado y que han repercutido en la mejora de la imagen turística de los centros históricos latinoamericanos. Así lo demuestran las actuaciones de iluminación y eliminación de cableado en la ciudad vieja de Santo Domingo en República Dominicana. Esta pequeña iniciativa supuso una mejora de la seguridad turística y un incremento de los flujos nocturnos sobre áreas históricas que no eran transitadas por visitantes (AECID, 2007). Algo similar sucedió con los inmuebles intervenidos en la ciudad colombiana de Popayán, sirviendo de aliciente para su reconocimiento turístico $(\mathrm{AECl}, 2002)$.

Por otro lado, y como se apuntaba con anterioridad, el empleo y los ingresos por turismo son considerados como otros efectos indirectos del programa, con una incidencia clara en la población residente (AECID, 2015). Empero, no existen investigaciones que permitan cuantificar este resultado y las aproximaciones a partir de las referencias estudiadas son exiguas y redundan sobre la incorporación laboral de los estudiantes de las Escuelas Taller.

Como igual se mencionó, desde sus primeras etapas, el programa recomendaba incluir capacitaciones en turismo, especialmente en las Escuelas Taller ubicadas en los centros históricos con mayor trayectoria en el ámbito. Al respecto, se aconsejaba que estos cursos incidieran en los parámetros de un desarrollo sostenible y comunitario, pero a la par, pudieran cubrir las necesidades de las profesiones turísticas más demandadas por los mercados laborales locales (AECID, 2015).

Es por ello, por lo que el alojamiento, la gastronomía, la guianza o las artesanías han sido los ámbitos de mayor incidencia. Además, estas profesiones permiten la integración de colectivos de gran vulnerabilidad, aspecto que destaca la cooperación española. También lo relaciona con la mejora de la calidad de los servicios turísticos y una disminución de la informalidad turística, como ha podido suceder en el Valle del

${ }^{6}$ Como se ha mencionado con anterioridad, algunos centros históricos ya eran destinos turísticos importantes antes de las intervenciones de la Cooperación Española, como es el caso de Salvador de Bahía, Cuzco o Antigua Guatemala. Otras ciudades con un desarrollo turístico de importancia eran Cartagena de Indias, Cuenca o Santo Domingo, mientras que la AECID reconoce que, en los casos de Suchitoto, Comayagua o Colón, el turismo podría ser una actividad económica que potenciar (AECID, 2003). 
Colca (AECID, 2012). En algún caso, como los cursos de guías de la Escuela Taller de Bogotá, han llegado a ser apoyados por el Instituto Distrital de Turismo (IDT), e incluso se han ampliado a otros sectores demandantes de formación en turismo como es el caso de botones, dependientes comerciales o taxistas. De esta forma, se enfatiza en la capacidad de formar en conocimientos turísticos básicos a las personas que tienen el primer contacto con los visitantes (AECID, 2015). La artesanía y gastronomía también permiten integrar el patrimonio cultural dentro de las ciudades históricas, mediante la recuperación de oficios y cocinas tradicionales, y la posibilidad de establecer negocios locales. Lo anterior, por ejemplo, se evidencia con la "filigrana momposina", que actualmente es un icono turístico de la ciudad histórica colombiana, producto de la recuperación de esta técnica a través de cursos ofertados en la escuela taller de la localidad (AECID, 2015).

Pero, y como también señala la AECID, junto con los establecimientos de artesanías, gastronomía o incluso pequeños alojamientos, las intervenciones del Programa de Patrimonio Cultural han podido tener una incidencia sobre efectos más negativos, como la implantación de franquicias internacionales de restauración $(\mathrm{AECl}, 2002)$. Otras situaciones indeseables que se señalan en las fuentes analizadas se relacionan con esas escenografías turísticas ya mencionadas o con procesos de especulación inmobiliaria como podría haber sucedido en Antigua o Santo Domingo (AECl, 2002).

En cualquier caso, no existen estudios ni investigaciones que arrojen datos más concluyentes sobre el impacto turístico del programa español, si bien la consideración del turismo como un área de complementariedad del programa y los nuevos cambios realizados en sus últimas etapas incitan a atender de manera más explicita las relaciones que, en este ámbito, se puedan desprender de las intervenciones futuras.

\subsection{El turismo desde la visión institucional de los actores intervinientes}

Como se ha podido evidenciar en este análisis de los resultados, tanto desde la teoría como desde la práctica, existe una evidente relación entre turismo cultural, centros históricos latinoamericanos y el programa de la cooperación española en Patrimonio cultural. Sin embargo, como se señala al principio de este texto, no siempre desde los actores intervinientes esas conexiones son tan claras o deseadas, e incluso a veces, generan contradicciones y problemas en la propia gestión.

Desde los organismos municipales y supramunicipales encargados de las funciones turísticas de los centros históricos latinoamericanos existe una tendencia a valorar al patrimonio urbano latinoamericano como un valor turístico estratégico. No obstante, a pesar de los efectos positivos que pueda haber, desde la gestión no son ajenos a problemas como la pérdida de identidad social o la desigual distribución de los beneficios generados por el turismo cultural. Al respecto, destaca la respuesta del exdirector general de la OMT para la región de "Las Américas", para quien, "turismo y cultura nunca están reñidos con la adecuada preservación histórico - artístico, sino todo lo contrario. El turismo pone en valor y da a conocer esos valores y permite contribuir con recursos para que se reinviertan en su preservación" (comunicación personal, 19 de julio de 2017).

En todo caso, cuando a los gestores turísticos se les pregunta por las acciones que en patrimonio cultural urbano latinoamericano desempeñan los organismos internacionales y las agencias de cooperación como la AECID, la mayor parte desconoce esta labor. De este modo, se observa una relación en cuanto al tamaño del centro histórico, y por ello, ciudades como Cartagena de Indias, Cuenca o Arequipa llegan a establecer nexos entre las Escuelas Taller y las intervenciones patrimoniales. Así mismo, por otro lado, ciudades más grandes y urbanas como Lima, apenas reconocen a estos centros por sus labores formativas. Tampoco se evidencia interrelación alguna por parte de otras instituciones con intervención regional, ya que como prosigue el exdirector para las América de la OMT,

La UNESCO se articula con la OMT, exclusivamente en la relación Madrid - París, si bien con relación a las ciudades patrimonio no hacemos acciones en concreto. Nosotros, con la UNESCO, lo que intentamos es unir esfuerzo para que turismo y cultura no estén enfrentados, sino que se puedan complementar. Por eso tratamos de persuadir al PNUD, por ejemplo, la necesidad de entender que el turismo es siempre un instrumento de desarrollo. (comunicación personal, 19 de julio de 2017). 
Estas palabras, que muestran esa tradicional antipatía por parte del sector cultural, se encuentran reforzadas por las manifestaciones de la responsable de la UNESCO para la región andina latinoamericana,

\begin{abstract}
Si hablamos de turismo porque hay que tenerlo en cuenta, pero tampoco satanizarlo. Con una buena regulación no se sale de control. A todo el mundo le gusta tener turistas porque ganan más dinero. Tengo colegas que dicen que no se puede sacar nada bueno del turismo, pero yo discrepo de esa opinión pues yo creo que si hay cosas que puede dar. Muchos sitios se han conservado porque tienen cosas que se puede ofrecer al turista, pero otra cosa es que eso se haya salido de las manos. Pero si puede ser un incentivo para la conservación y es necesario tenerlo en cuenta en su justa medida. (comunicación personal, 19 de septiembre de 2017).
\end{abstract}

Así, para la responsable de la UNESCO, debe existir una gran comunicación e información de los efectos que el turismo cultural puede generar en los centros histórico. Esta labor debe realizarse, principalmente, con las comunidades que viven en estos espacios urbanos y que, según ella, deben tener la libertad de poder decidir no querer "que sus barrios sean turísticos". También debe extrapolarse a todos los actores vinculados con el sector turístico y cultural, puesto que, como señala la portavoz de la UNESCO, en el territorio latinoamericano existen duplicidades de proyectos a partir del uso turístico del patrimonio cultural,

Todo el mundo quiere dar dinero para el turismo (...) En Tiahuanaco hay cinco proyectos (de cooperación) todos haciendo lo mismo y todos pensando en el turismo. Es un tema de moda, y las autoridades no tienen un filtro para decir ya estamos trabajando esto aquí (...) No solamente es el turismo y aceptar cualquier proyecto que venga porque es dinero y trae dinero. Hay que saber que se quiere y cómo se quiere. Los nacionales y los locales deberían de controlar esto. (comunicación personal, 19 de septiembre de 2017).

Además, incide en recordar que el dinero procedente de la cooperación internacional en patrimonio cultural debe reorientarse hacia el beneficio de los habitantes de los centros históricos. Solamente, y de manera secundaria, los proyectos se deben hacer pensando en los visitantes, con la excepción de que el turismo sea el objetivo principal de la intervención, si bien, aún en este caso, deviene fundamental transmitir unos alcances y unas expectativas realistas.

Aun cuando la visión del organismo internacional aboga por una visión al menos no excluyente del turismo cultural, los responsables del programa de la cooperación española presentan una opinión más ambivalente. La posición más crítica la ofrece la jefa adjunta del departamento de cooperación y promoción cultural de la AECID,

Preferiríamos que a estos lugares no fuese el turismo, que tiene una tendencia a ridiculizar (...) Yo creo que el turismo podría ser un beneficiario indirecto, pero muy cuidadoso. Porque me parece un negocio cuya inmediatez en directamente proporcional al daño que puede producir. El turismo rápido lo que hace es destrozar. Llega a un pobre pueblo y lo arrasa. El turismo es un depredador. (comunicación personal, 11 de julio de 2017).

Esta mirada podría resumir la consideración de muchos gestores culturales hacia la labor del turismo en los espacios patrimoniales. No obstante, como el resto de los actores entrevistados vinculados con la $A E C I D$, reconoce que el patrimonio es una herramienta para el desarrollo económico y la conversión de bienes culturales en atractivos turísticos. A tal efecto, como reconocen desde el departamento de Cooperación y Promoción Cultural: "claro que forma parte de las marcas turísticas, mira el convento de Santo Domingo en Cartagena de Indias, que es un icono y fue una intervención nuestra".

En esta misma línea coinciden otros responsables del programa. Para el responsable del Programa de Escuelas Taller para Latinoamérica, tales instituciones han favorecido el turismo por su contribución para poner en valor edificios, espacios públicos, pero también parques, en tanto que "son intervenciones sobre los entornos y eso contribuye al turismo, pero creo que también es un efecto secundario" (comunicación personal, 11 de julio de 2017)

Otra voz similar es la del responsable del programa de patrimonio en Ecuador, para quien programas como las Escuelas Taller tienen una relación clara con el turismo, aunque no exista reconocimiento de esta labor por parte de los turistas, 
La recuperación que se plantea desde la AECID genera turismo, especialmente, turismo histórico y turismo patrimonial, aunque siempre dependerá igualmente del contexto interno. En todo caso, el trabajo de la Alcaldía, junto con la labor de la AECID, ha permitido que Quito sea más visitado y que se prioricen las acciones en los barrios más denigrados, en donde también se han incrementado el flujo turístico. Algunos hoteles como el hotel Casa Plaza o Gangotona, a través de la recuperación de San Francisco, ha tenido una influencia perceptible en el incremento de la afluencia turística. (comunicación personal, 10 de junio de 2017).

Esta consideración vendría no solo a refrendar la vinculación directa a través de las intervenciones patrimoniales, sino también su repercusión en la actividad turística indirecta. Así mismo, el resto de entrevistados sugieren, con sus respuestas, que el centro histórico como el resto de los actores intervinientes son un aspecto fundamental para instrumentalizar turísticamente el programa. De esta manera, responsables de diferentes Escuelas Taller señalan la importancia que están adquiriendo los cursos de turismo, especialmente cuando las intervenciones cada vez son menores $\mathrm{y}$, por tanto, la demanda de cursos vinculados con la construcción está decayendo. En este sentido, las Escuelas Taller de Cuenca y Arequipa vislumbran en el turismo una oportunidad para la continuidad y sostenibilidad misma de sus respectivas escuelas. Otras, por el contrario, incluso van más allá, como el caso de Cartagena de Indias, cuya función trasciende a la gestión del patrimonio mundial,

\footnotetext{
Hay que recordar que en la última etapa se recibe el encargo de gestionar las fortificaciones y ahí se relaciona la Escuela Taller con el turismo. No es solo el mantenimiento de las fortificaciones sino un plan de la Escuela para la intervención y puesta en valor (...) con recorridos culturales en las murallas, con actividades, jornadas libres de entrada al castillo, actividades para los niños. Incluso, trabajamos con guías turísticos, o temas como las tarifas o las negociaciones con los cruceros (comunicación personal, 26 de junio de 2016).
}

Esta última aportación, realizada por el exdirector de la Escuela Taller de Cartagena de Indias, verificaría la capacidad de actuar como un actor de la cooperación internacional dentro de la gestión turística de los centros histórico. Aunque se trata de un caso excepcional, denota la capacidad de colaboración y de integración de la entidad dentro del territorio y de los ámbitos cultural y turístico. Una opinión local que contrasta, como se ha podido apreciar con las voces más institucionales del programa.

\section{Conclusiones}

El presente texto ha tratado de poner de manifiesto las diferentes formas en las que la cooperación española en patrimonio ha podido intervenir en el turismo de los centros históricos latinoamericanos. El análisis de los distintos contextos, fuentes y actores ha permitido establecer que si bien la cooperación no puede ni debe sustituir la labor de la gestión turística y/o patrimonial, sí que inciden notablemente en la reconversión de bienes culturales en atractivos turísticos.

Esta relación causa-efecto es perceptible desde la propia mención del uso económico del patrimonio cultural con el que se dio casi origen al programa y que se enfatizó especialmente durante sus primeras etapas. Al respecto, existe una evidente inclusión de términos que hacen una referencia indirecta al turismo, tal y como es el caso de "rentabilidad económica", "valor productivo" o "contribución privada del aprovechamiento patrimonial". En todo caso, y como se apunta, el programa se orienta cada vez más hacia un enfoque primordialmente comunitario, en el que el ascenso del turismo como sector de complementariedad por parte de los planes directores de la agencia española permite seguir determinando relaciones futuras.

Además, tanto por parte de las iniciativas internacionales como de las bilaterales, parece inevitable que la puesta en valor del patrimonio cultural urbano repercuta en el turismo. Tanto si este se integra dentro de los lineamientos como si no se contempla, cualquier iniciativa que permita un uso y acceso a los bienes culturales está posibilitando que parte o todo su público sea de carácter turístico. En el caso concreto de la cooperación española, las acciones de restauración, y especialmente las de adecuación de espacios públicos y privados, provocan una incidencia en la mejora de los entornos que incide en el incremento del atractivo turístico. Como señalan los responsables del programa, ciertos inmuebles han podido adquirir la categoría 
de iconos y/o hitos turísticos de estas ciudades, como es el caso del Convento de Santo Domingo en Cartagena de Indias o el de San Francisco en Quito.

También se han podido verificar estas interacciones en las Escuelas Taller, ya sea en su posición como centros formativos capaces de ofrecer cursos de capacitación en turismo -en ocasiones, dirigidos hacia colectivos vulnerables-, o en la responsabilidad de gestión ciertos inmuebles con vistas hacia su activación turística. Si bien ambos aspectos son reseñables para esta investigación, el primero permite pensar que la población local pueda ser beneficiaria directa, tanto por la generación de empleos como de ingresos.

De hecho, la integración de las comunidades resulta fundamental, además de por su condición como destinatarios del programa -tal y como redundan sus responsables-, por su importancia en el desarrollo de procesos participativos que se pueden orientar hacia la gobernanza turística. En este sentido, si las intervenciones tienen efectos sobre la capacidad de incrementar el turismo, las comunidades residentes de los centros históricos deben conocer tales circunstancias y sus posibles alternativas. Así mismo, en lo referente a disponer de capacidad de decisión e intervención durante todas las etapas de la ejecución de los proyectos. El desconocimiento de estas acciones debe evitarse también en el ámbito de la gestión turística, pues el reconocimiento de la cooperación internacional puede promover estrategias en el ámbito turístico. Igualmente, la inclusión de los actores turísticos, al menos, como observadores de estas iniciativas, permite evitar esa duplicidad manifiesta por los entrevistados, lo cual puede ser contemplado como un mecanismo de control para evitar tendencias acaparadoras. Así, y como ha quedado demostrado, una mayor obtención de recursos financieros no supone una solución per se a las necesidades locales.

No obstante, estos procesos pueden reducir la desconfianza de los actores patrimoniales sobre el turismo. Para ello, el conocimiento de los alcances reales de la relación entre cooperación, patrimonio y turismo debe ser más estudiado. Eso reduciría los recelos mostrados desde la visión institucional de los actores entrevistados. De hecho, conviene señalar cómo el turismo tiene mejor consideración entre los responsables locales del programa que respecto de aquellos actores con mayor rango. De este modo, se puede pensar en que la correcta aplicación en terreno del programa se acompaña de mayores y mejores sinergias.

En conclusión, el turismo pareciera ser contemplado como un aspecto secundario de la cooperación internacional en patrimonio, aun cuando sus alcances son múltiples y en algunos casos capaces de generar resultados positivos. En aquellos casos en los que se evidencia un trabajo conjunto por parte de diferentes actores procedentes del ámbito patrimonial y turístico, los efectos igualmente resultan positivos y establecen posibilidades que demandan un análisis diferenciado. El no querer visibilizar esta relación no impide que el turismo se desarrolle a partir de la intervención internacional en los centros históricos, sin embargo, su conocimiento sí que capacita al turismo y al patrimonio como instrumentos de desarrollo. Lo anterior, incluso permitiendo una autogestión por parte de las comunidades y entes locales cuando la cooperación internacional se retire de estos espacios patrimoniales y abandone su condición de actor interviniente en el sistema turístico.

\section{Referencias bibliográficas}

AECI (1999): Programa de Preservación del Patrimonio Cultural de Iberoamérica. Madrid: Agencia Española de Cooperación Internacional.

AECID (2002): Programa de Patrimonio Cultural de la Cooperación Española. Madrid: Agencia Española de Cooperación Internacional y R\&R (material multimedia).

- (2007): P>D Patrimonio para el Desarrollo: situación a noviembre de 2007. Madrid: Agencia Española de Cooperación Internacional para el Desarrollo.

- (2007a): Estrategia de Cultura y Desarrollo de la Cooperación Española. Madrid: Agencia Española para la Cooperación Internacional al Desarrollo.

- (2007b): Las Escuelas Taller en su Laberinto: Nuevos Enfoques y Desafíos: Resumen seminario interno de reflexión. Madrid: Agencia Española de Cooperación Internacional para el Desarrollo.

- (2009): Programa Patrimonio para el desarrollo. Madrid: Dirección de Relaciones Culturales y Científicas.

- (2010): Programa P>D Patrimonio para el Desarrollo. Madrid: Agencia Española de Cooperación Internacional al Desarrollo. 
— (2011): Programa P>D Patrimonio para el Desarrollo. Memoria. Madrid: Agencia Española de Cooperación Internacional al Desarrollo.

-(2012): Evaluación de 9 proyectos del programa Patrimonio para el Desarrollo en el marco del Programa de Cooperación Hispano Peruano (2007-2011). Madrid: Agencia Española para la Cooperación Internacional al Desarrollo y Agencia Peruana de Cooperación Internacional.

- (2013): Programa ACERCA. Madrid: Agencia Española de Cooperación Internacional para el Desarrollo.

- (2015): 25 años cooperando con América Latina y el Caribe. Vol. I. Conclusiones Encuentros. Madrid: Agencia Española de Cooperación Internacional para el Desarrollo.

Ahsworth, G. (1999): "Culture, tourism and cities: the inseperable triangle", en Matwijow, B. eds: Heritage and Development of Historic Cities. 138-148. Groningen: Universidad de Groningen.

Ballart, J. (2002): El patrimonio histórico y arqueológico: valor y uso. Barcelona: Ariel.

BID (2001): Una obra en marcha: el Banco Interamericano de Desarrollo y la protección del patrimonio cultural. Washington: Banco Interamericano de Desarrollo.

Brewer-Carias, A. R. (2008): El modelo urbano de la ciudad colonial y su implantación en Hispanoamérica. Bogotá: Universidad Externado de Colombia.

Carrión, F. (2007): Financiamiento de los centros históricos de América Latina y el Caribe. Quito: FLACSO.

Chueca-Goitia, F. (1968): Breve Historia del Urbanismo. Barcelona: Gustavo Gili.

De Terán, F. (2004): "Turismo, arquitectura y urbanismo: contextos históricos culturales de su relación", en: Actas del IV Congreso Fundación DOCOMOMO Ibérico: 129-140. Valencia.

Del Arenal, C. (2011): Política exterior de España y relaciones con América Latina: iberoamericanidad, europeización y atlantismo en la política exterior española. Madrid: Ediciones AKAL.

Gascón, J. y Canadá, E. (2005): Viajar a todo tren. Turismo, desarrollo y sostenibilidad. Barcelona: Icaria.

Gómez-Pallete, A. (2014). "El patrimonio como instrumento de desarrollo local. La actuación de la AECID en materia de puesta en valor del patrimonio", en Guridi, R., Ibáñez, J. y Vela, F. coord.: Proyectar la memoria: criterios y estrategias para la intervención, restauración y gestión del Patrimonio Cultural Iberoamericano: 147-152. Madrid: Rueda.

González-Varas, I. (1999): Conservación de Bienes Culturales. Teoría, historia, principios y normas. Madrid: Catedra.

González, F. y Morales, S. (2009): Ciudades efímeras: transformando el turismo urbano a través de la producción de eventos. Barcelona: Editorial UOC

Gutiérrez, R. (2014): "Repensando el patrimonio desde América Latina", en Zingoni, J. y Pinassi, A. comp.: Gestión del patrimonio urbano: textos de cátedra: 63-80. Buenos Aires: Ediuns.

Gutman, M. y Hardoy, J. (1992): Impacto de la urbanización en los centros históricos de Iberoamérica. Madrid: Editorial Mapfre.

Hiernaux, D. y González, C. I. (2015): "Patrimonio y turismo en centros históricos de ciudades medias. ¿Imaginarios encontrados?", URBS. Revista de Estudios Urbanos y Ciencias Sociales, 5 (2): 111-125.

Jordán, L. y Duval, D.T. (2009): "Heritage Management and tourism in the Carribbean", en Timothy, D. y Nyaupane, G. eds: Cultural Heritage and Tourism in the Developing World. A regional perspectiva: 186209. Londres: Routledge.

López, L. (1999): "El papel de los centros históricos en los sistemas urbanos", en Bernal, B. coord.: Ciudad histórica y realidad urbana: 43-49. Burgos: Universidad de Burgos-La Caixa.

Menchero, M. (2019): Centros Históricos, Cooperación Internacionaly Turismo: Programa Patrimonio Cultural y Escuelas Taller para la región andina. Madrid: Universidad Rey Juan Carlos. (Tesis doctoral).

- y Ríos, J. (2020): "Programa Patrimonio Cultural y Escuelas Taller: análisis de la cooperación cultural española en América Latina y sus centros históricos", Revista Aportes. Revista de Historia Contemporánea, 35 (102): 149-183.

Morère, N. y Perelló, S. (2014): Turismo cultural, patrimonio, museos y empleabilidad. Madrid: Escuela de Organización Industrial.

Muntal, S. (2003): "Ciudades y centros históricos de América Latina y el Caribe. Algunas consideraciones", en: // Encuentro sobre Manejo y Gestión de Centros Históricos. La Habana.

Nel-lo, M. y Pérez, M. (2015): "La Cooperación Internacional de la Universidad en el ámbito del turismo", Pasos Online, 13 (1): 983-1001. https://doi.org/10.25145/j.pasos.2015.13.068

OMT (2005): City Tourism \& Culture; the European Experience. Madrid: Organización Mundial del Turismo. 
Orbasli, A. (2002): Tourists in Historic Towns: Urban Conservation and Heritage Management. Nueva York: Taylor \& Francis.

Prats, L. (1997): Antropología y patrimonio. Barcelona: Ariel.

Pulido, J. (2015): "Los actores del turismo cultural", en Pulido, J. coord.: Turismo Cultural. 143-169. Madrid: Síntesis.

Pulin, F. (1993): "La Carta de Veracruz en las ciudades históricas", en: Actas del Congreso Internacional de Urbanismo y Conservación de Ciudades Patrimonio de la Humanidad. 61-64. Mérida: Asamblea de Extremadura.

Quinto Centenario (1991): Programa de revitalización de centros históricos de Iberoamérica. Madrid: Quinto Centenario.

Richards, G. (2008): "Cultural Tourism: Global and Local Perspectives", Journal of Cultural Economics, 32 (3): 231-236.

Sanz, N. (1996): "Para una economía del patrimonio: la entropía y los bienes de interés cultural", Complutum, 2: $261-272$.

Schlüter, R. (1998): "Tourism development in Latin American", Annals of Tourism Research, 20: 364-367.

Tresserras, J. (2002): "El turismo cultural en países en vías de desarrollo", en: / Congreso Internacional del Turismo Cultural. Salamanca.

UNESCO (1969): La protección del patrimonio cultural de la Humanidad. Lugares y monumentos. París: UNESCO.

— (2014): Plan de acción para América Latina y el Caribe (2014 - 2024). París: UNESCO.

\section{Breve CV de la autora}

Miriam Menchero Sánchez es Doctora en Turismo y profesora a tiempo completo en la Facultad de Empresa y Comunicación de la Universidad Internacional de La Rioja - UNIR (España). 